\title{
THE EFFECTS OF NEIGHBORING BUILDINGS ON THE INDOOR WIRELESS CHANNEL AT 2.4 AND $5.8 \mathrm{GHz}$
}

\author{
Do-Young Kwak*, Chang-hoon Lee*, Eun-Su Kim*, Seong-Cheol Kim*, and Joonsoo Choi** \\ * Institute of New Media and Communications, Seoul National University, Republic of Korea \\ ** Department of Computer Science, Kookmin University, Republic of Korea \\ E-MAIL: \{dykwak, lchjsa, eskim, sckim\}@maxwell.snu.ac.kr, jschoi@kookmin.ac.kr \\ TEL: +82-2-880-8421, FAX: +82-2-885-4459
}

Keywords: Indoor wireless propagation channel, ray-tracing technique, neighbouring buildings effects

\begin{abstract}
The effects of neighboring buildings (NB's) on the indoor wireless channel are examined both in time and space domain at $2.4 \mathrm{GHz}$ and $5.8 \mathrm{GHz}$ band using the computer simulation of radio wave propagation based on ray-tracing technique. The NB's in the apartment environments have a considerable effect on the channel characteristics, such as the exponents of path loss, mean excess delay, rms delay spread time, coherence bandwidth, and angle dispersion of received rays. Also the effects are shown to be different according to the frequency band.
\end{abstract}

\section{Introduction}

WLAN (Wireless Local Area Network) based on the standards of IEEE $802.11 \mathrm{a} / \mathrm{b} / \mathrm{g}$ equipped with multiple antenna array is one of the appropriate solutions to support a high data rate home network services. In most suggested indoor wireless channel, it is assumed that the excess delays of multipath signals do not exceed several hundreds nsec (about 300 nsec). But when WLAN service is offered in the apartment complex where the buildings are fairly close to each other, this assumption is invalid because of the existence of signals with a long excess delay. These signals propagate via the reflection at the exterior walls of the NB's. In this paper, we examine the effects of NB's on the indoor wireless channel at 2.4 and $5.8 \mathrm{GHz}$ using the computer simulation of radio wave propagation based on the raytracing technique. Its accuracy is verified by comparing with wideband measurements at $5.8 \mathrm{GHz}$. The examination is focused on the analysis of channel parameters in power, time, frequency, and space domain.

\section{Channel model}

The multipath components (MPC's) in indoor environment are arriving in clusters as suggested in the Saleh-
Valenzuela channel model[4]. The expanded SalehValenzuela spatial-channel model including azimuth AoA information[5] was suggested as follows

$$
h(t, \theta)=\sum_{l=0}^{L} \sum_{k=0}^{K} \beta_{k l} e^{j \phi_{k l}} \delta\left(t-T_{l}-\tau_{k l}\right) \delta\left(\theta-\Theta_{l}-\omega_{k l}\right)
$$

, where $L$ represents the number of clusters, $K$ the number of MPC's within each cluster, $e^{j \phi_{k l}}$ phase of each MPC, $\mathrm{T}_{1}$ cluster arrival time, $\tau_{\mathrm{kl}}$ ray arrival delay time, $\Theta_{\mathrm{l}}$ cluster angle and $\omega_{\mathrm{kl}}$ ray angle, respectively. In this equation, angle parameters $\Theta_{\mathrm{l}}$ and $\omega_{\mathrm{kl}}$ are set to relative angles with respect to the direct path between Tx and $\mathrm{Rx}, \beta_{k l}$ is the amplitude of each MPC and generally assumed to be a Rayleighdistributed random variable, whose mean squared value is modelled by

$$
\overline{\beta_{k l}^{2}}=\overline{\beta^{2}\left(T_{l}, \tau_{k l}\right)}=\overline{\beta^{2}(0,0)} e^{-T_{l} / \Gamma} e^{-\tau_{k l} / \gamma}
$$

, where $\Gamma$ and $\gamma$ represent cluster decay time constant and ray decay time constant, respectively. And the sum of $\overline{\beta_{k l}^{2}}$ represents the average power for a CW signal.

The channel parameters in time domain such as mean excess delay $\bar{\tau}$ and rms delay spread $\sigma_{\tau}$ are given as follows[3]

$$
\begin{gathered}
\bar{\tau}=\frac{\int_{-\infty}^{\infty} t s(t) d t}{\int_{-\infty}^{\infty} s(t) d t} \\
\sigma_{\tau}=\sqrt{\frac{\int_{-\infty}^{\infty}(t-\bar{\tau})^{2} s(t) d t}{\int_{-\infty}^{\infty} s(t) d t}}
\end{gathered}
$$


, where $s(t)\left(=\left|\int h(t, \theta) d \theta\right|^{2}\right)$ is the power delay profile of the channel. And the coherence bandwidth can be determined from the obtained frequency coherence obtained using the power delay profile as following equation[3]

$$
R_{H}(\Delta f)=\int_{-\infty}^{\infty} R(t) \cdot e^{-j 2 \pi \Delta f t} d t
$$

, where $R(t)(=E[s(t)])$ is the expectation value of the power delay profile. In this study, the coherence bandwidth is defined as the bandwidth over which the value of frequency coherence is above 0.9 .

The channel parameters in space domain such as mean angle of arrival $\bar{\theta}$ and rms angle spread $\sigma_{\mathrm{As}}$ can be similarly calculated as $\bar{\tau}$ and $\sigma_{\tau}$ as follows[6]

$$
\begin{gathered}
\bar{\theta}=\frac{\int_{-\pi}^{\pi} \theta s(\theta) d \theta}{\int_{-\pi}^{\pi} s(\theta) d \theta} \\
\sigma_{A S}=\sqrt{\frac{\int_{-\pi}^{\pi}(\theta-\bar{\theta})^{2} s(\theta) d \theta}{\int_{-\pi}^{\pi} s(\theta) d \theta}}
\end{gathered}
$$

, where $s(\theta)\left(=\left|\int h(t, \theta) d t\right|^{2}\right)$ is the angle delay profile of the channel

\section{Environments, Measurements, and Com- puter simulations}

The wideband channel measurements at $5.8 \mathrm{GHz}$ and simulation of radio wave propagation using ray-tracing technique at 2.4 and $5.8 \mathrm{GHz}$ are carried out in the apartment environment. High-rise apartment building is one of the most popular residence types in Asian countries, especially in South Korea. The apartment buildings in South Korea have some unique characteristics such that they are not more than $20 \mathrm{~m}$ in its thickness and usually $10 \sim 20$ stories high and some similar buildings are located in row in an apartment environment. Each floor of the building consists of several dwelling units having the same structure. There is no corridor in the middle of the building, which being different from office buildings. The apartment building in this study has 20 stories and each floor consists of 4 dwelling units having 3 bedrooms and 1 living room. 3 similar buildings are located in row with the distance of $57 \mathrm{~m}$ between them. The exterior walls are made of concrete $\left(\varepsilon_{\mathrm{r}}=7-\mathrm{j} 0.3\right)$ and glass $\left(\varepsilon_{\mathrm{r}}=5.4\right)$, while the interior walls are made of concrete and brick $\left(\varepsilon_{\mathrm{r}}=4.4-\mathrm{j} 0.031\right)$.

The wideband channel measurements using PNcorrelation method[2] are carried out at $5.8 \mathrm{GHz}$ in the $5^{\text {th }}$ floor of the building. Delay time resolution of MPC is $20 \mathrm{~ns}$ and one impulse response at one Rx position is obtained per $164 \mu$ s, which corresponds to 2 sequence periods of Msequence used in measurement system. The bandwidth of the signal is $50 \mathrm{MHz}$. Txs and Rxs are located in the middle building of the 3 buildings and 2 Tx locations and 16 and 17 Rx locations according to Txs are selected as shown in Figure 1. Tx and Rx antenna heights are $1.7 \mathrm{~m}$ and $1.6 \mathrm{~m}$, respectively.

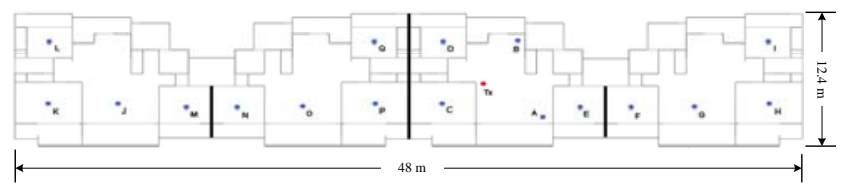

(a)

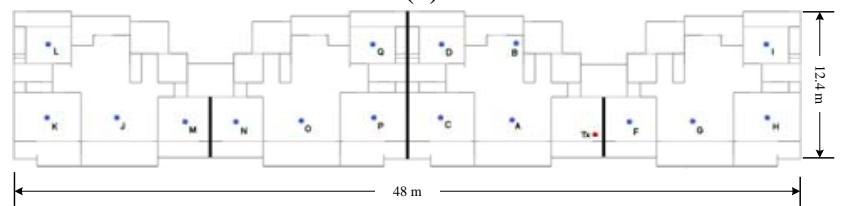

(b)

Figure 1 Building footprint of the $5^{\text {th }}$ floor with Tx/Rxs positions: (a) Tx 1 (b) Tx 2

The computer simulation of radio wave propagation using ray-tracing technique based on pin-cushion method is carried out with the same $\mathrm{Tx}$ and $\mathrm{Rx}$ positions in measurement at 2.4 and $5.8 \mathrm{GHz}$. The ray paths carrying power of $-30 \mathrm{~dB}$ or less relative to the strongest ray at each receiver are neglected because these rarely contribute to the total received power. The comparisons of measured data and simulated data for two Rx positions are shown in Figure 2. The dotted line and the thin solid line describe the measured results using PN-correlation method and the impulse response based on the amplitude of each received ray, respectively. The thick solid line denotes the pulse response obtained by calculating the coherent sum using the amplitude and the phase of each MPC in order to compare the PNcorrelation measured result directly. The wideband comparisons show that the predicted results are agreed well with the measured results.

\section{Results and Analysis}

In the previous research[1], the NB's had major effect not on the received signal power but on the rms delay spread. But in this apartment environment such that the buildings are fairly close (about several tens meters), the MPC's, which undergo the reflection at the exterior wall of the NB's have a considerable effects on the channel characteristics. When the 


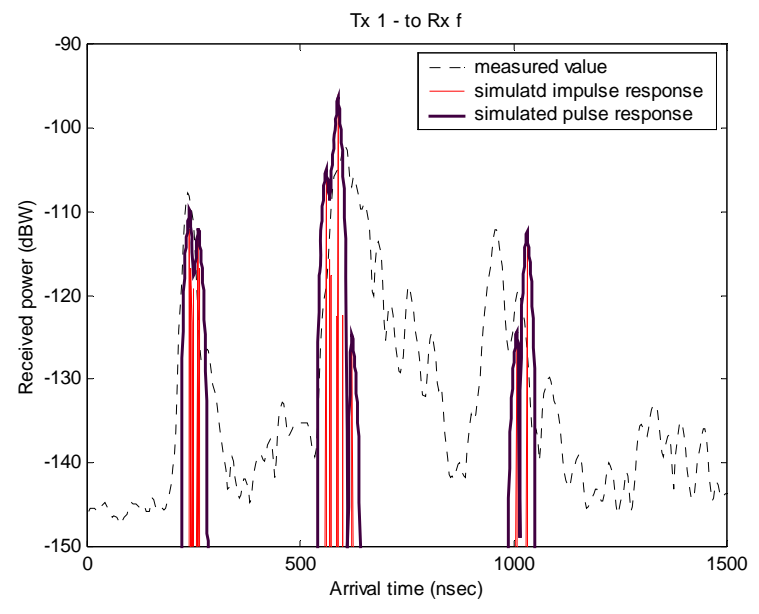

(a)

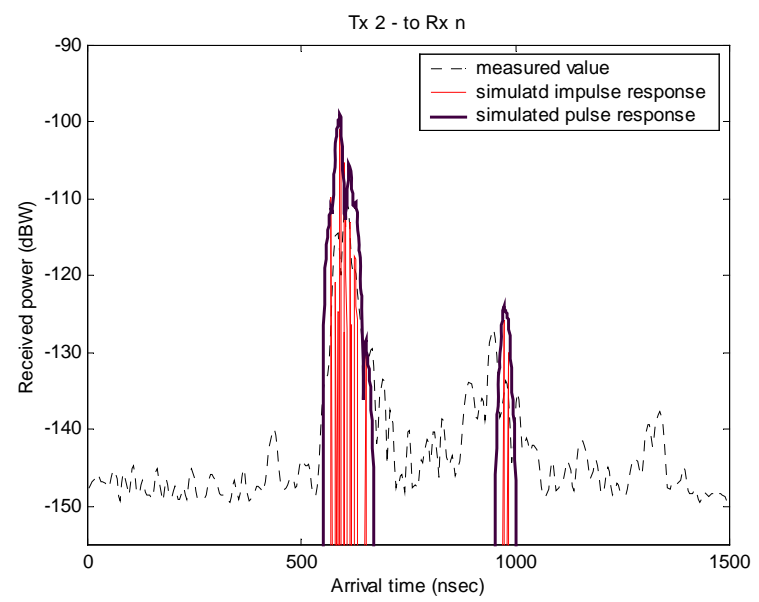

(b)

Figure 2 Comparison of measurements and simulations at 5.8 GHz: (a) Tx1-Rx f (b) Tx2-Rx n

Rxs are close to the Tx in the range of about $10 \mathrm{~m}$, the effects of the arrived MPC's via the reflection at the exterior wall of the NB's can be negligible because the power of MPC's via reflection and transmission within building are too strong. But as the Rxs are apart from the Tx, the power portion of MPC's via the reflection at the NB's to the total received power is large. Therefore these signals have a considerable effect on the wireless channel characteristics.

Firstly, the average power for a CW signal at the Rxs has relatively large value when we considered the NB's as compared with those when we do not considered the NB's as shown in Figure 3. The differences of the exponents of path loss are 3.2 and 5.4, at $2.4 \mathrm{GHz}$ and $5.8 \mathrm{GHz}$, respectively.

Secondly, the delay time parameters, which influence on the system performance, also have large differences according to whether the NB's exist or not as shown in Figure 4. The differences of averaged mean excess delay and rms delay spread are $91.4 \mathrm{nsec}$ and $77.4 \mathrm{nsec}$ at $2.4 \mathrm{GHz}$ and 105.8

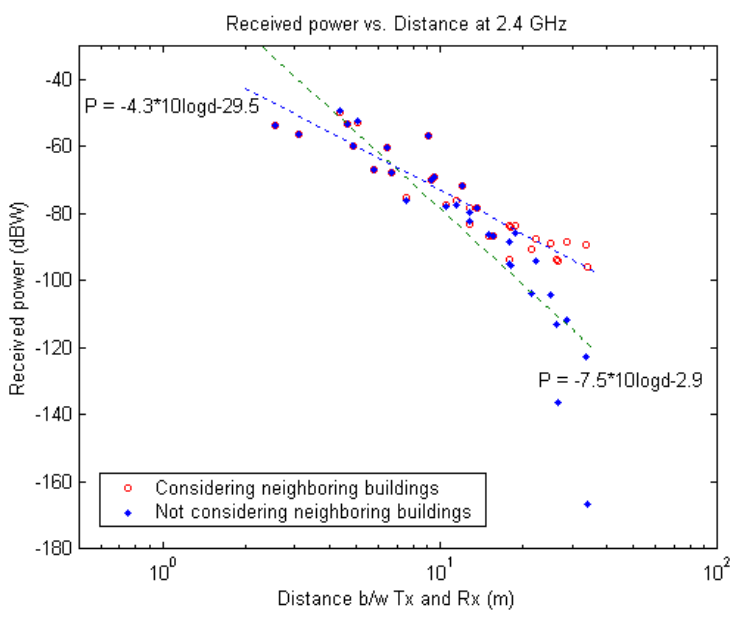

(a)

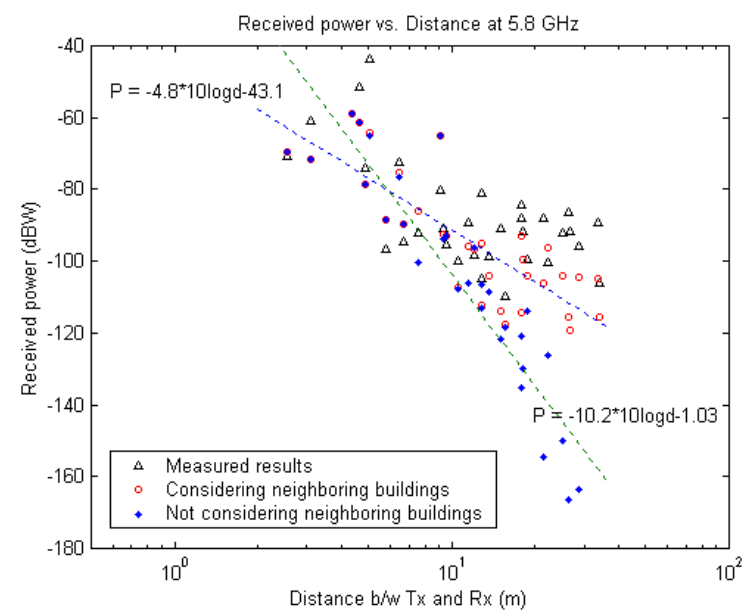

(b)

Figure 3 Received CW powers at the Rxs: (a) $2.4 \mathrm{GHz}$ (b) $5.8 \mathrm{GHz}$

nsec and 56.6 nsec at $5.8 \mathrm{GHz}$, respectively. Therefore MPC's with long delay time (more than $800 \mathrm{nsec}$ ), which deteriorate WLAN system performance, are more likely to be appeared when the fairly closed NB's are existed. Also in this case, the coherence bandwidth is small (lower than 1 $\mathrm{MHz}$ ) as shown in Figure 5 and the channel is possible to be a frequency selective fading channel. The statistics of channel parameters in time and frequency domain are described in Table 1.

Finally, the power azimuthal profiles (PAP's) of MPC's at downlink (Tx-to-Rx) are examined. The averaged PAS over 33 Tx-Rx pairs for two cases (considering NB's and not considering NB's) are plotted in Figure 6. The powers of each ray are normalized to the strongest ray power at each $\mathrm{Tx}-\mathrm{Rx}$ pair in order to obtain PAS. In other words, the strongest ray power at each Tx-Rx pair is set to $1 \mathrm{~W}$ and the relative power of other rays to $1 \mathrm{~W}$ are calculated and the PAS's are obtained using the relative power of MPC's. 


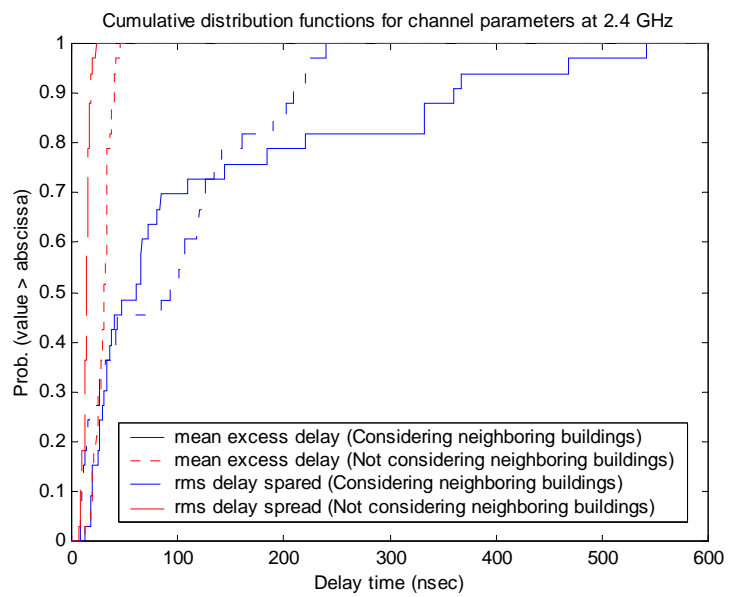

(a)

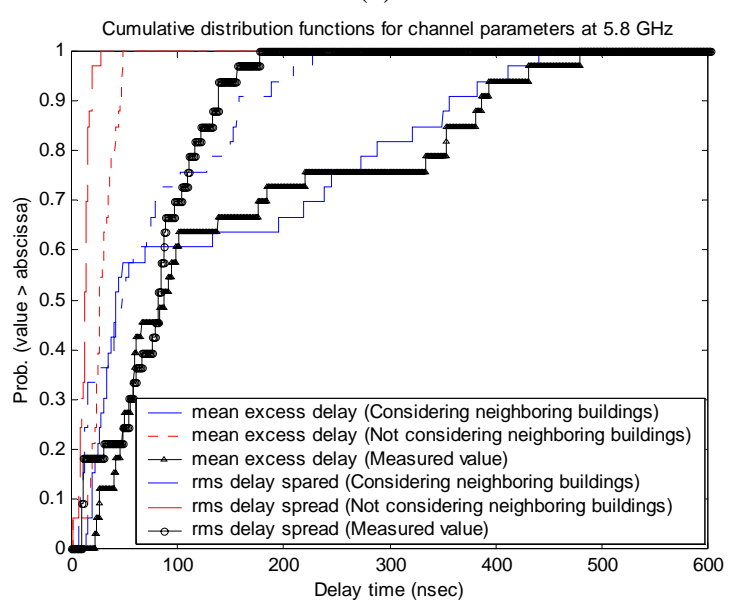

(b)

Figure 4 Time delay parameters:(a) $2.4 \mathrm{GHz}$ (b) $5.8 \mathrm{GHz}$

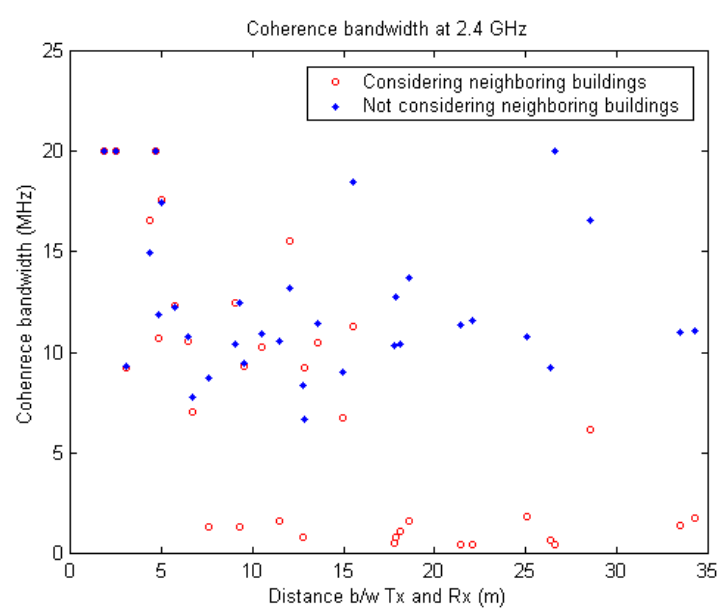

(a)

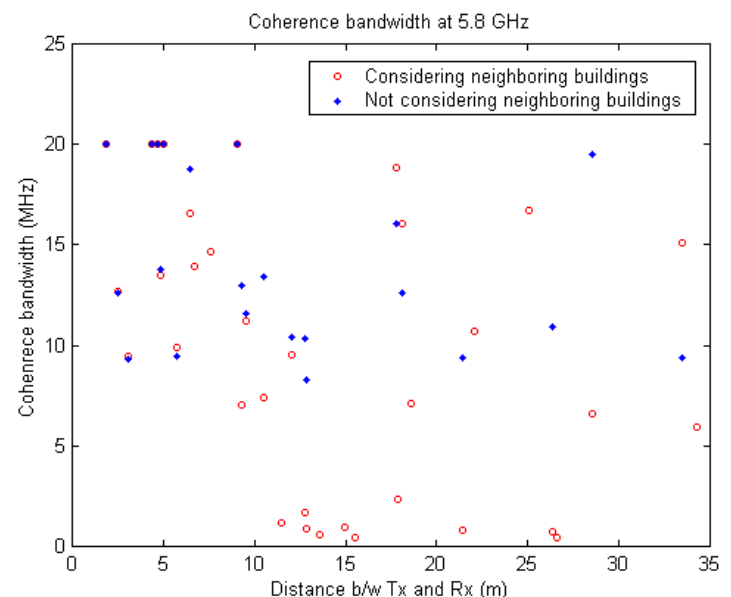

(b)

Figure 5 Coherence bandwidth at Rxs: (a) $2.4 \mathrm{GHz}$ (b) 5.8 $\mathrm{GHz}$

\begin{tabular}{|c|c|c|c|c|c|}
\hline \multirow{2}{*}{\multicolumn{2}{|c|}{ Parameters }} & \multicolumn{2}{|c|}{ Considering NB’s } & \multicolumn{2}{|c|}{ Not considering NB's } \\
\hline & & Avg. & Std dev & Avg. & Std dev \\
\hline \multirow{3}{*}{$\begin{array}{c}2.4 \\
\mathrm{GHz}\end{array}$} & $\begin{array}{l}\text { Mean excess } \\
\text { delay (nsec) }\end{array}$ & 120.47 & 143.25 & 29.05 & 7.37 \\
\hline & $\begin{array}{c}\text { Rms delay } \\
\text { spread (nsec) }\end{array}$ & 90.44 & 74.25 & 13.02 & 3.29 \\
\hline & $\begin{array}{c}\text { Coherence } \\
\text { bandwidth } \\
(\mathrm{MHz})\end{array}$ & 7.40 & 6.53 & 12.43 & 3.73 \\
\hline \multirow{3}{*}{$\begin{array}{c}5.8 \\
\mathrm{GHz}\end{array}$} & $\begin{array}{l}\text { Mean excess } \\
\text { delay (nsec) }\end{array}$ & 135.39 & 140.59 & 29.55 & 9.88 \\
\hline & $\begin{array}{l}\text { Rms delay } \\
\text { spread (nsec) }\end{array}$ & 69.01 & 64.23 & 12.44 & 4.42 \\
\hline & $\begin{array}{c}\text { Coherence } \\
\text { bandwidth } \\
(\mathrm{MHz})\end{array}$ & 9.79 & 6.97 & 14.03 & 4.37 \\
\hline
\end{tabular}

Table 1 Statistics of channel parameters in time domain

They have very large value at the direct path between Tx and Rx when the NB's are not considered. But when the NB's are considered, the PAP's have considerable values at the direction to the NB's. The mean angle of arrival and rms angle spread based on the averaged PAS with NB's are $6.28^{\circ}$ and $75.1^{\circ}$ at $2.4 \mathrm{GHz}$ and $-11.4^{\circ}$ and $79.9^{\circ}$ at $5.8 \mathrm{GHz}$, respectively. Meanwhile the mean angle of arrival and rms angle spread based on the averaged PAS without NB's are small as $1.88^{\circ}$ and $69.1^{\circ}$ at $2.4 \mathrm{GHz}$ and $5.6^{\circ}$ and $57.8^{\circ}$ at $5.8 \mathrm{GHz}$, respectively. Therefore it is expected the MIMO channel capacity, which depends on the independency of the characteristics of MPC's, especially angle spread, will be increased when there exist fairly closed NB's. 


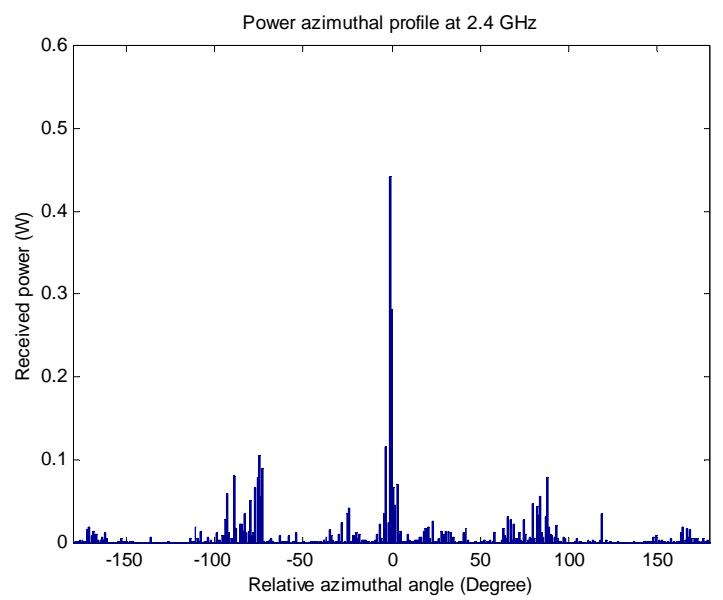

(a)

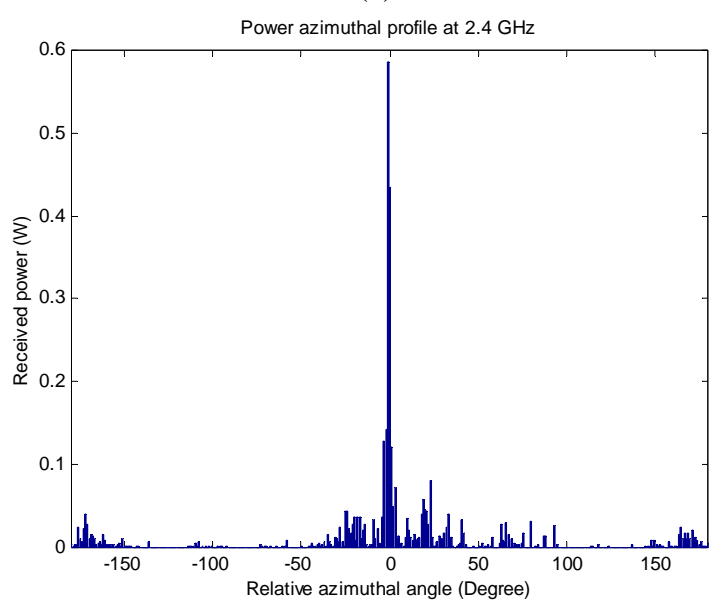

(b)

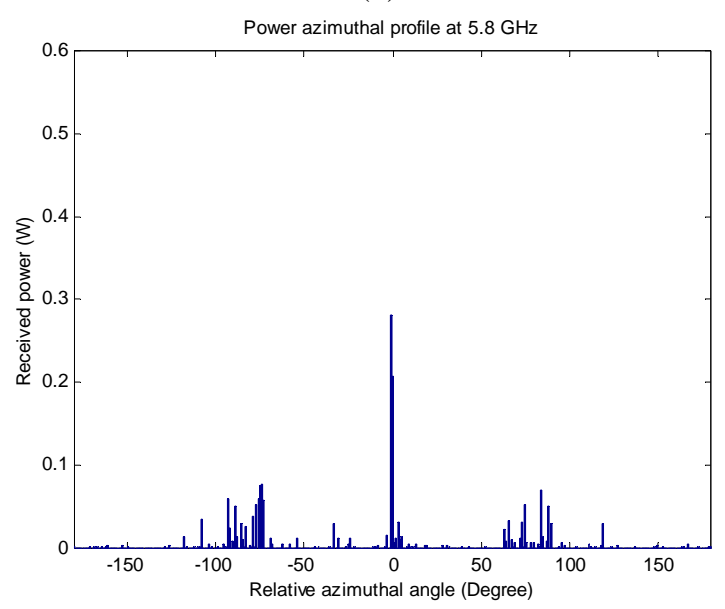

(c)

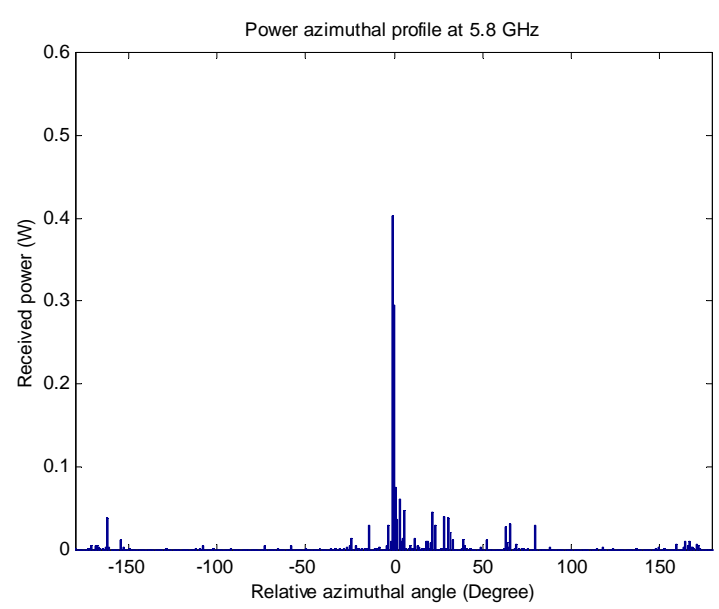

(d)

Figure 6 Power azimuthal profiles at Rxs: (a) at $2.4 \mathrm{GHz}$, considering NB's (b) at $2.4 \mathrm{GHz}$, not considering NB's (c) at $5.8 \mathrm{GHz}$, considering NB's (d) at $5.8 \mathrm{GHz}$, not considering NB's

The NB's have distinct effects on channel characteristics in both frequencies, but the effects are more apparent at 5.8 $\mathrm{GHz}$ than at $2.4 \mathrm{GHz}$. The reason is that when rays undergo transmission or reflection at walls, the excess losses are large at high frequency. Therefore the rays via the reflection at the walls of NB's have more contributions to the channel characteristics at $5.8 \mathrm{GHz}$ than $2.4 \mathrm{GHz}$.

\section{Conclusion}

The effects of NB's on channel characteristics are examined at two frequencies $(2.4 \mathrm{GHz}$ and $5.8 \mathrm{GHz})$ in the apartment environments. The NB's decrease the exponents of path loss of about $3 \sim 5$ and coherence bandwidth of about 5 $\mathrm{MHz}$. And these increase mean excess delay of about 100 nsec, rms delay spread of about $50 \sim 80$ nsec. The mean angle of arrival and rms delay spread are also affected by NB's. Moreover these effects are shown to be more apparent at higher frequency because of the differences of wall characteristics such as reflection, transmission, and diffraction characteristics in different frequency band.

\section{Acknowledgement}

This work was supported in part by Brain Korea 21 Project in 2005 and in part by ITRC.

\section{References}

[1] G.E. Athanaasiadou; A. R. Nix, and J. P. McGeehan, "A new 3D indoor ray-tracing propagation model with particular reference to the prediction of power and delay spread,” in Proc. IEEE Intl. Symp. Personal, Indoor 
and Mobile Radio Communications, vol. 3, pp. 27-29, (1995).

[2] D. C. Cox, "Delay Doppler characteristics of multipath delay spread and average excess delay for $910 \mathrm{MHz}$ urban mobile radio paths,” IEEE Trans. Antennas Propag., vol. 20, pp. 625-635, (1972).

[3] S. C. Kim, H. L. Bertoni, and M. Stern, "Pulse propagation characteristics at $2.4 \mathrm{GHz}$ inside buildings," IEEE Trans. Veh. Technol., vol. 45, pp. 579-592, (1996).

[4] A. A. M. Saleh and R. A. Valenzuela, "A statistical model for indoor multipath propagation,” IEEE J. Select. Areas Commun., vol. 5, pp. 128-137, (1987).

[5] Q. Spencer, B. D. Jeffs, A. Jensen, and A. L. Swindlehurst, "Modeling the statistical time and angle of arrival characteristics of an indoor multipath channel," IEEE J. Select. Areas Commun., vol. 18, pp. 347-360, (2000).

[6] K. Yu, Q. Li and M. Ho, "Measurement investigation of tap and cluster angular spreads at $5.2 \mathrm{GHz}$," IEEE Trans. Antennas Propag., vol. 53, pp. 2156-2160, (2005). 